\title{
Flexible Defense Succeeds Creative Attacks!-A Simulation Approach Based on Position Data in Professional Football
}

\author{
Daniel Memmert', Jonas Imkamp1, Jürgen Perl² \\ ${ }^{1}$ German University of Sport Science, Cologne, Germany \\ ${ }^{2}$ University of Mainz, Mainz, Germany \\ Email: j.imkamp@dshs-koeln.de
}

How to cite this paper: Memmert, D., Imkamp, J. and Perl, J. (2021) Flexible Defense Succeeds Creative Attacks!-A Simulation Approach Based on Position Data in Professional Football. Journal of Software Engineering and Applications, 14, 493-504.

https://doi.org/10.4236/jsea.2021.149029

Received: July 6, 2021

Accepted: September 13, 2021

Published: September 16, 2021

Copyright $\odot 2021$ by author(s) and Scientific Research Publishing Inc. This work is licensed under the Creative Commons Attribution International License (CC BY 4.0).

http://creativecommons.org/licenses/by/4.0/

(c) (i) Open Access

\begin{abstract}
Introduction: The key to success is finding the perfect mixture of tactical patterns and sudden breaks of them, which depends on the behavior of the opponent team and is not easy to estimate by just watching matches. According to the specific tactical team behavior of "attack vs. defense" professional football matches are investigated based on a simulation approach, professional football matches are investigated according to the specific tactical team behavior of "attack vs. defense." Methods: The formation patterns of all the sample games are categorized by SOCCER $\odot$ for defense and attack. Monte Carlo-Simulation can evaluate the mathematical, optimal strategy. The interaction simulation between attack and defense shows optimal flexibility rates for both tactical groups. Approach: A simulation approach based on 40 position data sets of the 2014/15 German Bundesliga has been conducted to analyze and optimize such strategic team behavior in professional soccer. Results: The results revealed that both attack and defense have optimal planning rates to be more successful. The more complex the success indicator, the more successful attacking player groups get. The results also show that defensive player groups always succeed in attacking groups below a specific planning rate value. Conclusion: Groups are always succeeding. The simulation-based position data analysis shows successful strategic behavior patterns for attack and defense. Attacking player groups need very high flexibility to be successful (stay in ball possession). In contrast, defensive player groups only need to be below a defined flexibility rate to be guaranteed more success.
\end{abstract}

\section{Keywords}

Strategy, KPI, Offensive and Defensive Dynamics, Ball Contact, Ball Possession, Passes 


\section{Introduction and Theoretical Framework}

The almost unknown Union Berlin in the German Bundesliga ranked $7^{\text {th }}$ in the 2020/2021 Bundesliga league table, while Hertha BSC, the well-known Berlin soccer team, was close to relegation. A reasonable explanation of the sudden success of Union Berlin could be their specific style of playing, where flexible actions of defensive player groups with the ball suddenly break into periods of stereotype defensive action patterns. In contrast to this, Hertha BSC shows the efforts of the creative action of offensive player groups. The key to success is to find the perfect mixture of tactical patterns and sudden breaks freedom. This balance of strategies, of course, depends on the behavior of the opponent team and is not relatively easy to estimate by just watching games (Memmert \& Raabe, 2018 [1]). To shed light on the perfect balance of professional football flexibility, we provide and test a simulation framework based on big data (positional data of the players and the ball).

Work in the simulation of positional data is closely related to trajectory prediction and theory on multi-agent simulation (e.g., Wellman, 2006 [2]). Once you train your model to predict movements, you can simulate all game actors (Tuyls et al., 2020 [3]). This action is usually called "ghosting" in the literature (Lowe, 2013 [4]). This approach is first used in soccer or basketball (Maksai, Wang, \& Fua, 2016 [5]; Le, Carr, Yue \& Lucey, 2017 [6]; Le, Yue, Carr, \& Lucey, 2017 [7]). However, so far, no attempts were made on position data simulations themselves.

This study analyzes the defensive- and attacking lines of Bundesliga teams by Monte Carlo Simulation to determine the best possible flexibility for both.

\section{Simulation-Based Approach on Tactical Patterns in Football}

Some teams win using "Tiki Taka," and other teams win by breaking "Tiki Taka" by fast counter attacks. The main goal of our simulation approach is to understand which offensive and defensive strategic concepts and tactical flexibility are successful in interacting with the opponent ones. To this aim, we analyze matches strongly reduced to actions and interactions of specific player groups (formations) like defense and offense. The constellations of the respective players, i.e., the types of appearance, are straightforward for tactical situations like ball contact, ball possession, and passing or the strategic background of interaction. Therefore, reduced to those formation interactions and tactical aspects, the strategic plans can be recognized and calculated, and optimized by simulation, using mathematical approaches like Monte Carlo simulation and game theory to generate optimal strategic patterns (Perl \& Memmert, 2018a [8], b [9]).

Such an optimal strategy consists typically of a variety of partial methods like, e.g., "conserving ball possession," "build attack," "fast attack," or "playing the ball directed to the goal" (Memmert \& Rein, 2018 [10]). Thus, while only a little data is available to calculate and decide what was victorious from the original 
match, an accurate simulation can generate lots of data to estimate what could have been successful. The result is a percentage distribution of the leading and remaining strategies.

However, for complex games like soccer with more than 20 interacting players, such a strategy distribution is much too complicated to get to work. It has to be simplified to player group-specific tactical variations. Therefore, Perl (2018 [11]) provides a simulation framework that generates a specific primary or "strict" strategy for just one type of success, e.g., space control, which gives room for situation-depending "flexible" variations. The number of generated flexible variations can be smaller or larger regarding the strict primary strategy, respectively defining a percentage degree of flexibility $\mathrm{F}$ against the remaining amount of MC-simulated actions that establishes the percentage degree of strictness S: S $+\mathrm{F}=100$. One example of a stringent space control strategy is the well-known "Tiki Taka." An example of a successful flexible counter-strategy is "fast counter-attacks."

For the first time, Perl, Imkamp, and Memmert (2021 [12]) tested this simulation framework by answering the question of whether "strictness" and "flexibility" are at all acceptable terms for characterizing strategic plans and tactical variations as well as their success. 40 positional data sets extracted from 10 professional soccer games were analyzed to find the original vital strategies, simulate the optimal primary strategy, and compare the values of strictness and flexibility. Moreover, soccer experts evaluated those results based on video analyses and confirmed the applicability of that simulation approach to professional Football.

Based on Perl et al. (2021 [12]), the current approach presents additional and more detailed results regarding the essential topics of flexible defense and creative attack in Football based on a simulation framework. In addition, we expand the study from Perl et al. (2021 [12]) by analyzing three performance indicators (ball contact, ball possession, and passes) in the simulation framework instead of one (ball contact).

\section{Method}

The positional data of the selected sample of $N=10$ matches from 2014/15 German Bundesliga was used for verification and evaluation using simulative and network-based process analysis. Football coaches (UEFA A-license) took the selected sample of the ten matches based on the teams' tactical orientation. Substitution effects and tactical formation bias were avoided by only using the first half of the matches (Memmert \& Raabe 2018 [1]). Each team in the sample played in an offensive formation with a four-man defensive line, two defensive midfielders, a central offensive midfielder, two wingers, and a forward, thus in a 4-2-3-1. The defensive formation of all teams is a 4-4-2. All success values (ball contact, ball possession, passes) for every simulated interaction of tactical player groups are averaged, and the threshold values are defined. This procedure is for Bundesliga team A and Bundesliga team B (both five games) and all three player groups. 
The intention is to simulate the interplay between the tactical groups of both teams. The tactical groups, defense, and attack are first determined. Then, the four-man defensive line players, $2,3,4$, and 5, are selected for the defense. Next, players 9,10 , and 11 were chosen for the tactical player group attack, and finally, the interaction of defense and attack were simulated in both directions. In this case, the goalkeeper is disregarded. Therefore, performance values for two tactical (defensive vs. attack) player groups of both teams of 10 matches result in a data set of $N=40$. The positions of the two tactical player groups are recorded per second and reduced to 20 distinct constellation types with the use of the SOCCER@ neuronal network (see for more details, Perl \& Memmert, 2011 [13]; Perl, Grunz, \& Memmert, 2013 [14]). The formation data of every team and tactical player group is trained on that artificial neural network to generate clusters of similar constellations during the training process, thus characterizing the constellation types by performance indicators. The interplay interaction of SOCCER $\odot$ is based on these trained formation types.

The actual game action is replaced by the interaction between these constellation types in the simulation. Several performance indicators (see Low et al., 2019 [15]), such as ball contact, space control, or pass-game, can be used to evaluate success (Memmert \& Raabe, 2018 [1]). The simulation of the performance indicators by combining attacking and defensive constellations is based on the previously mentioned clustering of constellations. Since only ball contacts are not a very complex indicator for success (Perl \& Memmert, 2017 [16]), this current study evaluates success by using "ball contact" in connection with "ball possession" and "passes" as one single overall performance indicator. Therefore, the total of 10 games was split into the two teams' raw positional data per match section, and each team's trajectories were divided into the attack and defensive constellation. Overall, we have $\mathrm{N}=40$ position data sets, three performance indicators, and two tactical groups per team of every selected match as a final result.

The core of the analysis consists of a comparison between the success-oriented recommendation that is derived from strategy optimization on the one hand and a Monte Carlo simulation of the actual tactical behavior on the other hand. The "planning rate" or "strictness" then is defined by the percentage of coincidence between essential and recommended behavior, i.e., the ratio with which the calculated primary strategy ("plan") is implemented in the match (for more details, see Perl et al., 2021 [12]). The complement to the planning rate $S$ is the flexibility rate $F: S+F=100$. The flexibility rate measures the percentage with which the primary strategy is deviated by tactical variations depending on the situation. A high planning rate describes a highly planned or stereotypical game that the opponent can easily recognize. A low planning rate leads to more flexible, tactical behavior. Too low planning rate values can lead to high flexibility or aimless conduct.

\section{Simulation Approach}

The simulation by SOCCER $\odot$ provides simulated success values of both teams in 
distribution tables in an $11 \times 11$ matrix. The $\mathrm{X}$-axis shows the planning rate of team B from 0 to 100 and its flexibility rate from 100 to 0 , both in steps of 10 . On the Y-axis, team A's planning rate and flexibility rate are also shown from 0 to 100 and 100 to 0 . The simulated numbers of successful events of the given success parameter (ball contact) per halftime are shown as the success values: The higher the value, the more successful.

Figure 1 shows the success values for the selected performance indicator (ball contact) and the planning rates on the left for team A and the right for team B. Every success value shows the simulated numbers of successful ball contacts for each team. The tables in the second row show both teams' positive or negative results as differences of the respective performance values depending on the individual planning rates. In the next step, the threshold values of the flexibilities are determined by the balance point of the optimal 0 -sum-distribution. The threshold values describe the line where the positive performance difference values change into negative values (for more detailed information, see Perl et al., $2021[12])$.

\section{Results}

The threshold evaluation of the simulated interaction between attack and defense for the three performance indicators, ball contact (Figure 2), ball possession (Figure 3), and passes (Figure 4), show a high dispersion demonstrated by

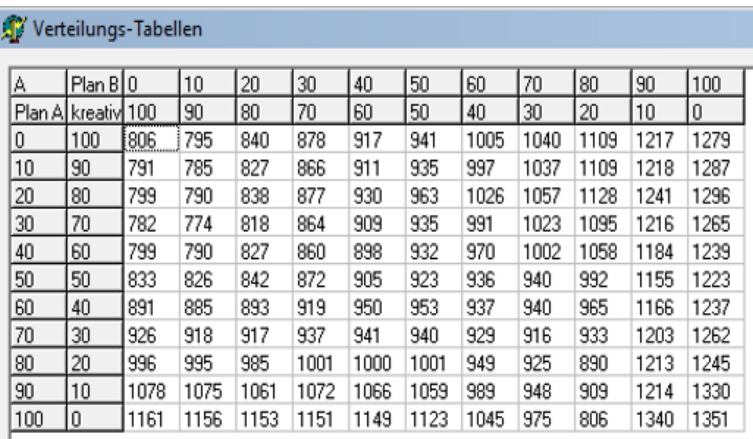

\begin{tabular}{|l|l|l|l|l|l|l|l|l|l|l|l|l|}
\hline B & Plan B & 0 & 10 & 20 & 30 & 40 & 50 & 60 & 70 & 80 & 90 & 100 \\
\hline PlanA & kreativ & 100 & 90 & 80 & 70 & 60 & 50 & 40 & 30 & 20 & 10 & 0 \\
\hline 0 & 100 & 755 & 738 & 739 & 773 & 841 & 907 & 962 & 1028 & 1099 & 1169 & 1246 \\
\hline 10 & 90 & 733 & 717 & 720 & 760 & 828 & 896 & 955 & 1018 & 1092 & 1160 & 1234 \\
\hline 20 & 80 & 773 & 761 & 761 & 788 & 840 & 900 & 953 & 1006 & 1078 & 1132 & 1213 \\
\hline 30 & 70 & 807 & 794 & 793 & 812 & 865 & 932 & 981 & 1037 & 1111 & 1169 & 1247 \\
\hline 40 & 60 & 860 & 849 & 850 & 874 & 923 & 982 & 1029 & 1081 & 1163 & 1201 & 1260 \\
\hline 50 & 50 & 928 & 914 & 927 & 951 & 998 & 1065 & 1120 & 1186 & 1266 & 1262 & 1309 \\
\hline 60 & 40 & 1001 & 987 & 1000 & 1018 & 1063 & 1119 & 1183 & 1242 & 1327 & 1274 & 1320 \\
\hline 70 & 30 & 1075 & 1069 & 1097 & 1110 & 1172 & 1230 & 1280 & 1340 & 1425 & 1290 & 1334 \\
\hline 80 & 20 & 1165 & 1154 & 1178 & 1186 & 1237 & 1292 & 1377 & 1424 & 1538 & 1330 & 1384 \\
\hline 90 & 10 & 1232 & 1227 & 1251 & 1267 & 1333 & 1382 & 1463 & 1523 & 1595 & 1367 & 1355 \\
\hline 100 & 0 & 1271 & 1282 & 1303 & 1315 & 1376 & 1430 & 1520 & 1625 & 1815 & 1311 & 1419 \\
\hline
\end{tabular}

\begin{tabular}{|c|c|c|c|c|c|c|c|c|c|c|c|c|}
\hline \multicolumn{4}{|c|}{ A.V.Matrix speichern } & & & & & & & & & \\
\hline$A \cdot B$ & $P \operatorname{lan} B$ & 0 & 10 & 20 & 30 & 40 & 50 & 60 & 70 & 80 & 90 & 100 \\
\hline Plan A & kreativ & 100 & 90 & 80 & 70 & 60 & 50 & 40 & 30 & 20 & 10 & 0 \\
\hline 0 & 100 & 51 & 57 & 101 & 105 & 76 & 34 & 43 & 12 & 10 & 48 & 33 \\
\hline 10 & 90 & 58 & 68 & 107 & 106 & 83 & 39 & 42 & 19 & 17 & 58 & 53 \\
\hline 20 & 80 & 26 & 29 & 77 & 89 & 90 & 63 & 73 & 51 & 50 & 109 & 83 \\
\hline 30 & 70 & .25 & .20 & 25 & 52 & 44 & 3 & 10 & .14 & .16 & 47 & 18 \\
\hline 40 & 60 & 61 & .59 & .23 & .14 & .25 & .50 & .59 & .79 & .105 & .17 & .21 \\
\hline 50 & 50 & .95 & .88 & .85 & .79 & .93 & .142 & .184 & .246 & .274 & .107 & .86 \\
\hline 60 & 40 & .110 & .102 & .107 & .99 & .113 & .166 & .246 & .302 & .362 & .108 & .83 \\
\hline 70 & 30 & .149 & .151 & .180 & .173 & .231 & .290 & .351 & .424 & .492 & .87 & .72 \\
\hline 80 & 20 & .169 & .159 & .193 & .185 & .237 & .291 & -428 & -499 & .648 & .117 & .139 \\
\hline 90 & 10 & .154 & .152 & -190 & -195 & .267 & .323 & -474 & .575 & -686 & .153 & .25 \\
\hline 100 & 0 & .110 & -126 & .150 & -164 & -227 & .307 & -475 & .650 & -1009 & 29 & -68 \\
\hline
\end{tabular}

\begin{tabular}{|c|c|c|c|c|c|c|c|c|c|c|c|c|}
\hline \multicolumn{4}{|c|}{ B.V-Matrix speichern } & & & & & & & & & \\
\hline$B-A$ & PlanE & 0 & 10 & 20 & 30 & 40 & 50 & 60 & 70 & 80 & 90 & 100 \\
\hline Plan A & kreati & 100 & 90 & 80 & 70 & 60 & 50 & 40 & 30 & 20 & 10 & 0 \\
\hline 0 & 100 & .51 & .57 & -101 & -105 & .76 & -34 & -43 & -12 & .10 & .48 & .33 \\
\hline 10 & 90 & .58 & 68 & .107 & -106 & .83 & -39 & -42 & -19 & .17 & .58 & .53 \\
\hline 20 & 80 & .26 & .29 & .77 & .89 & .90 & 63 & .73 & .51 & .50 & 109 & .83 \\
\hline 30 & 70 & 25 & 20 & .25 & .52 & .44 & -3 & .10 & 14 & 16 & .47 & .18 \\
\hline 40 & 60 & 61 & 59 & 23 & 14 & 25 & 50 & 59 & 79 & 105 & 17 & 21 \\
\hline 50 & 50 & 95 & 88 & 85 & 79 & 93 & 142 & 184 & 246 & 274 & 107 & 86 \\
\hline 60 & 40 & 110 & 102 & 107 & 99 & 113 & 166 & 246 & 302 & 362 & 108 & 83 \\
\hline 70 & 30 & 149 & 151 & 180 & 173 & 231 & 290 & 351 & 424 & 492 & 87 & 72 \\
\hline 80 & 20 & 169 & 159 & 193 & 185 & 237 & 291 & 428 & 499 & 648 & 117 & 139 \\
\hline 90 & 10 & 154 & 152 & 190 & 195 & 267 & 323 & 474 & 575 & 686 & 153 & 25 \\
\hline 100 & 0 & 110 & 126 & 150 & 164 & 227 & 307 & 475 & 650 & 1009 & .29 & 68 \\
\hline
\end{tabular}

Figure 1. Example screenshot of distribution tables created by SOCCERఠ. The X-axis shows the strictness of team B from 0 to 100 (planning rate), and the Y-axis, the strictness of team A. Top tables show simulated, successful event values for every flexibility combination of offense and defense for team A on the left and team B on the right. The bottom tables show successful event values of team A minus successful event values of team B for all flexibility combinations on the left and team B minus team A on the right. The red line marks the threshold. 
the standard deviation. These results were also found to analyze the attack, defense player groups of all Bundesliga Teams in the sample. The results for all three indicators show similar patterns. Figure 2 shows the threshold value curve of all ball contacts. Figure 3 shows the same for the indicator ball possession, and in Figure 4, the threshold values of the interaction simulation by SOCCER@ are demonstrated for the indicator passes.

The threshold results for each of the three given performance indicators start with low to mid-low values and increase by the higher planning rates of the attack. The threshold values are higher with the indicator ball contact than ball possession and pass. The more complex the indicators become, the more the threshold values align themselves to a linear curve.

\subsection{Success Indicator: Ball Contact}

SOCCER@ defines the performance indicator ball contact as an event in which coordinates of the ball are close to the coordinates of the nearest player. All ball contacts for the defense and the attack player groups are added and shown in distribution tables (Figure 1). All threshold values are based on the simulated positive ball contacts of both player groups. Thus, the indicator ball contact is the less complex indicator that SOCCER@ can evaluate.

In Figure 2, the threshold values for the performance indicator ball contact are shown. It shows the average threshold values of all ten matches with all

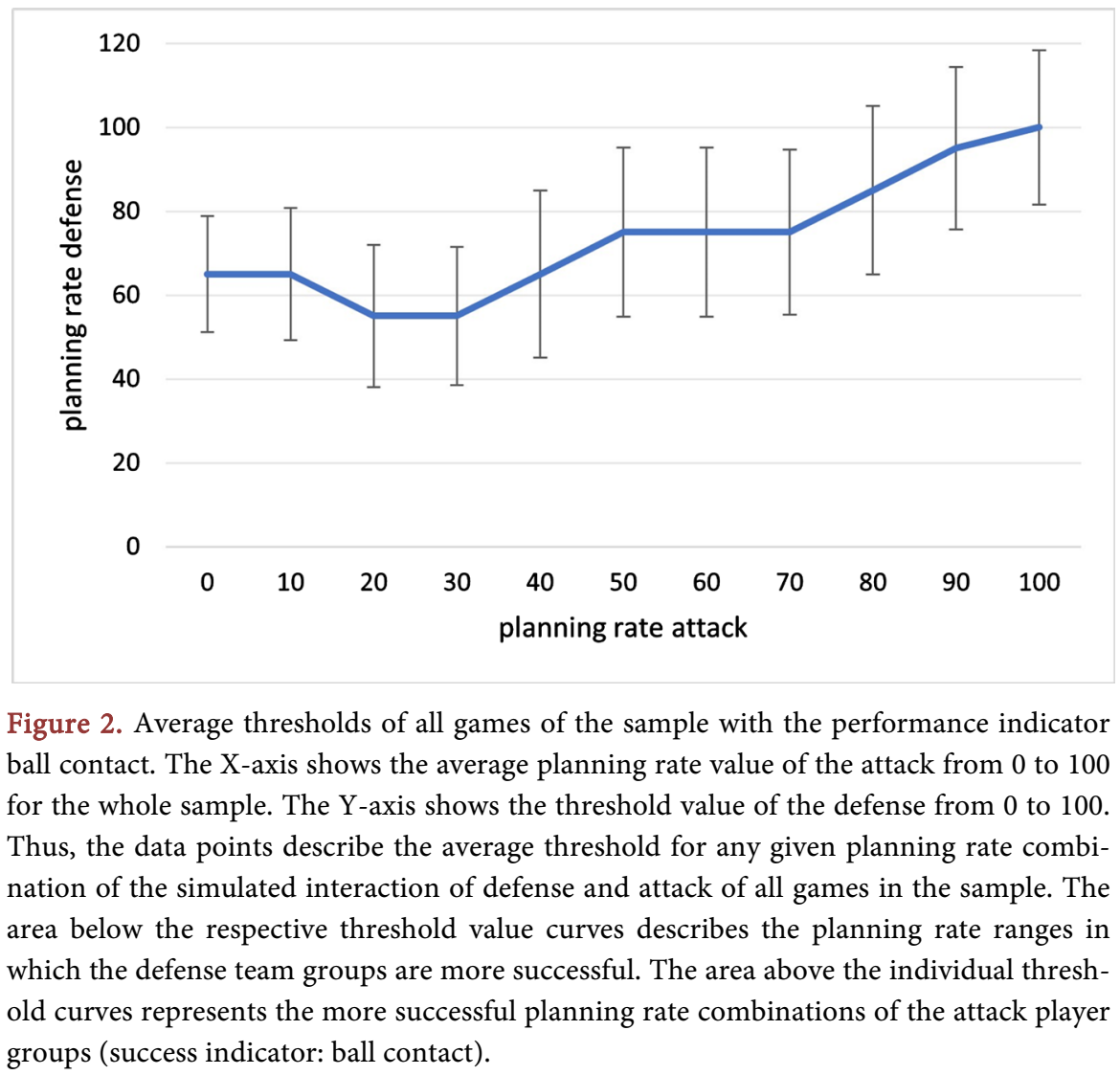


defense and attack player groups of all teams. It shows the successful attack ranges (above the threshold graph) and defense (below the threshold graph). Figure 3 and Figure 4, Figure 2 also offers the maximum value of 100 for the attack and defense planning rates. This can be explained by the lack of change in the match, which is positive for defensive player groups. Figure 2 shows that the optimal flexibility of an attack, depending on ball contacts, is between planning rates of 20 and 30. Even higher flexibility rates do not result in more success.

Very high planning rates (strictness) of the defense are the areas of more successful ball contacts of the attack. Defense player groups need, on average, a higher planning rate to gain more successful ball contacts than the attack. However, the success of a player group is not only influenced by its planning rate. Also, the interaction of both player groups and their planning rates is essential.

\subsection{Success Indicator: Ball Possession}

Ball possession is defined as a period of 2 seconds in which the coordinates of the closest player to the ball and the ball's coordinates are as relative as for ball contact. These successful ball possession events in the interaction of attack and defense are simulated by SOCCER $\odot$ and shown in distribution tables (Figure 1). The middle threshold values between positive ball possession values of the attack and the defense from all ten matches are shown in Figure 3.

Figure 3 shows that the optimal planning rate of an attack depending on ball

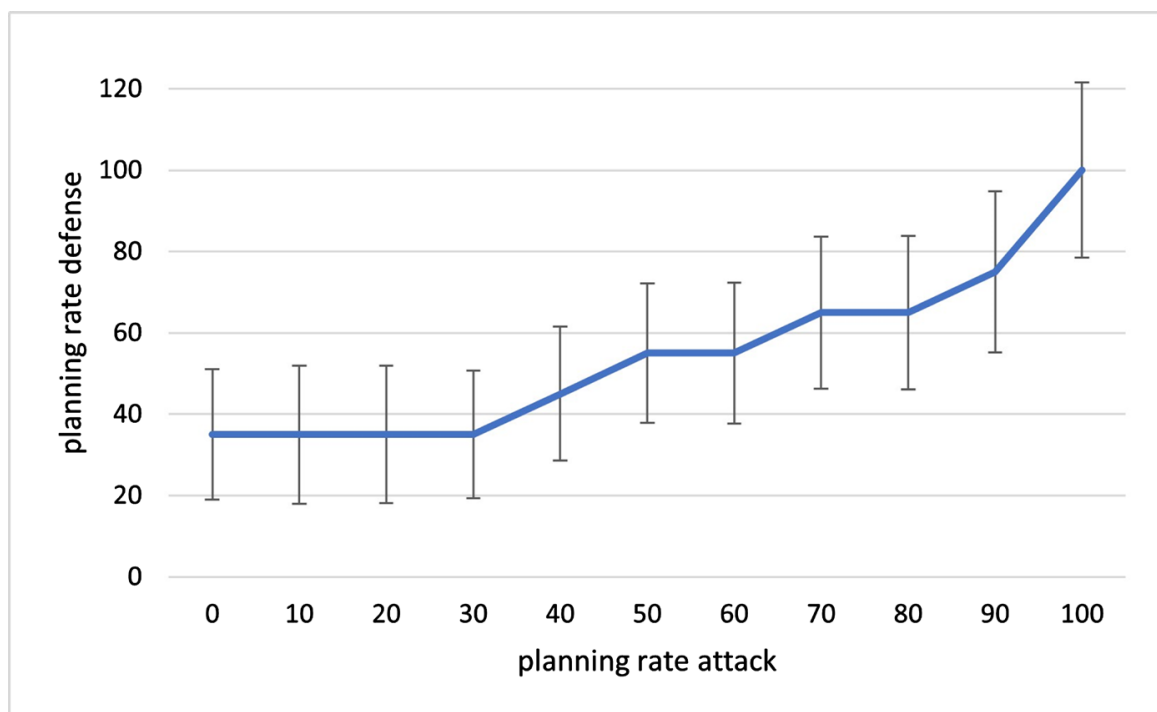

Figure 3. Average thresholds of all games of the sample with the performance indicator ball possession. The $\mathrm{X}$-axis shows the average planning rate value of the attack from 0 to 100 for the whole sample. The $\mathrm{Y}$-axis shows the threshold value of the defense from 0 to 100. Thus, the data points describe the average threshold for any given planning rate combination of the simulated interaction of defense and attack of all games in the sample. The area below the respective threshold value curves describes the planning rate ranges in which the defense team groups are more successful. The area above the individual threshold curves represents the more successful planning rate combinations of the attack player groups (success indicator: ball possession). 
possession lies between 0 and 30, with a planning rate range of the defense from 35 to 100 in which the attack succeeds the defense. The results are shown in Figure 3 also describe that defensive player groups have a planning rate below 35. They will be more successful in ball possession in every planning rate of the attack. The higher the planning rates of the attack, the higher the success rates of the defense get.

\subsection{Success Indicator: Pass}

The success indicator pass simulated by SOCCER $\odot$ is defined as a ball possession of the passing player before the pass and a successful ball possession of a different player of the same team after the pass. This indicator was detected by SOCCER $\odot$ adds the time of passing and position of the involved players. This indicator is the most complex indicator of ball contact, ball possession, and pass. The successful passes are simulated interactively by SOCCER $\odot$ for every given planning rate combination of attack and defense. These successful passing values are shown in distribution tables (Figure 1). The middle threshold values of all 10 matches are shown in Figure 4.

Figure 4 shows an optimal attack planning rate of 0 to 30 with a threshold value of 25 . That means the success range goes from defense planning rate 25 to 100. From attack planning rate 30 , the threshold values rise nearly as the planning rate rises. With a 100 attack and defense planning rate, the attack still has a

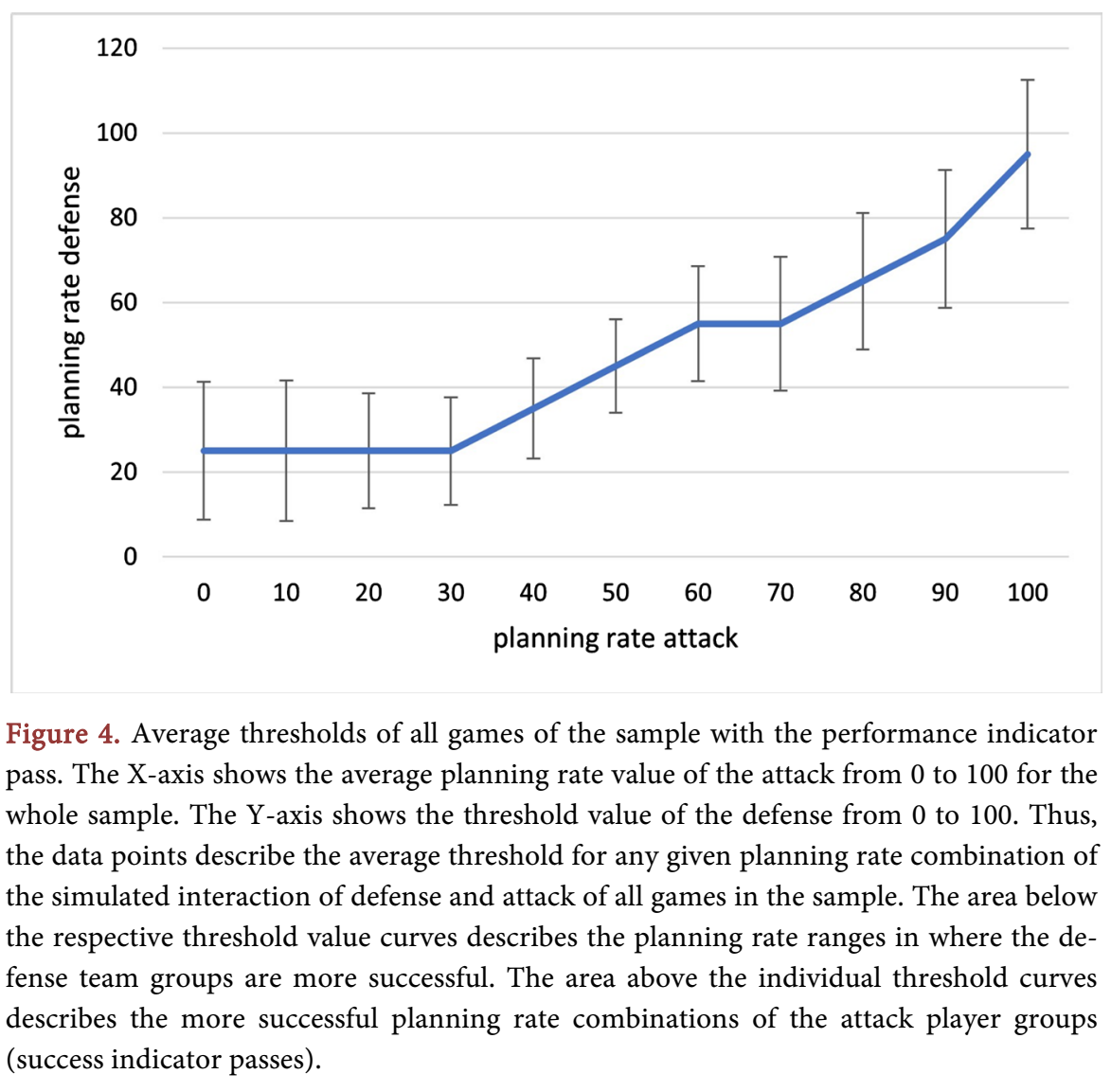


small success range of 5 .

The threshold values of the indicator pass show a similar curve as the threshold values of ball contact and ball possession. The main differences are the success ranges for optimal attack planning rates. The more complex the indicator gets, the more extensive the success goes for optimal attack planning rates. This can be the result of a lower count of detected events for more complex indicators. Fewer events lead to more extreme middle values. The theory can also explain these results that defending player groups can defend less complicated events of a flexible or creative attack. On the other hand, if the events get more complex and have the same high flexibility, it gets more challenging to defend.

\section{Discussion}

Notational analysis approaches aimed to determine the successful tactical behavior of different team members. Still, it could not investigate interaction processes in team strategies (Memmert \& Raabe, 2018 (1)) because it is narrowed to the already seen strategy and not the overall space of possibilities. Therefore, the main aim of this study was to establish a simulation approach in professional Football with a simulation of the tactical behavior "attack vs. defense" in soccer as a function of the strategic planning rate based on three fundamental performance indicators.

The results for all indicators show a similar threshold curve. The main difference is the threshold values at low attack planning rates. The more complex the indicator gets, the lower the planning rate values at low or optimal attack planning rates are. Thus, more complex events and low attack planning rates result in more extensive success ranges of the attacking player group.

This can be interpreted in different ways. The more complex an indicator gets, SOCCER@ will detect the more minor events. That can influence threshold values to more extreme values. It could also mean that if a complex event is played highly flexible or creative, it is more difficult to defend than events of lower complexity. Passes between highly flexible positioned attacking player groups seem to have high success. Based on this assumption, passing drills should be modified. Different positions in every trial could lead to more flexibility in the games. This could also train defense player groups to find the perfect balance between flexibility and strictness.

Results also show a very high success range from attack planning rate 0 to 100 at low planning rate values of the defense. At ball contact from 0 to 55, at ball possession from 0 to 35 , and passes from 0 to 25 . This means that defense player formations should play highly flexible. They will succeed against attack player groups no matter how flexible they are. For practical implications, the results can be summarized as follows: offensive player groups should play more flexible or creative tactical variations to stay in ball possession, whereas defensive player groups need the right balance between flexibility and strictness. The results for ball contacts show that defense formations with planning rates between 60 to 70 
are likely to be the more successful team. This changes with the indicator ball possession, where the defense is more successful with low planning rates no matter how the attack plays. The results of all indicators show that an optimal planning rate for an attack should be between 20 and 30 to be as successful as possible.

The practical effect of such approaches and measures can be checked utilizing exemplary concrete games. First, the teams become instructed about their tactical-strategic behavior and then set creative accents freely or controlled by simulation results. The first attempts for such approaches are the studies by Memmert et al. (2019 [17]) and Low et al. (2021 [18]). Both presented field experiments in an 11 vs. 11 soccer game set-up to investigate the impact of different formations (e.g., 4-2-3-1 vs. 3-5-2) and pressing strategies (high vs. low pressing) on tactical key performance indicators using positional data a controlled design. This demonstrated that an experimental positional data analysis paradigm is a valuable tool to develop theory-oriented models in performance analysis in team sports.

For this purpose, portable sensors (GPS tracking systems, see Baca et al., 2009 [19]) are needed to collect the position data of players from both teams in a field-based but controlled environment. Furthermore, by using several matches in the simulation, more reliable results will be achieved. Also, the increased number of matches can take better account of the variability and improve the teams' flexibility. This will lead to further development of theory-driven simulation models (Groom, Cushion, \& Nelson, 2011 [20]; Rein, Perl, \& Memmert, 2017 [21]) and incorporate existing talent and periodization models in simulation approaches.

In conclusion, high flexibility is the key to success in soccer, depending on the three indicators of ball possession: Monte Carlo-based analysis shows this leads to optimizing tactical processes. In particular, highly flexible defensive formations have an advantage over attacking formations. Finally, it shows that flexibility needs to be integrated in practice for every tactical part of the team to increase the overall performance.

\section{Conclusions}

The simulation-based position data analysis shows successful strategic behavior patterns for attack and defense. Attacking player groups need very high flexibility to be successful (stay in ball possession). In contrast, defensive player groups only need to be below a defined flexibility rate to be guaranteed more success.

The results for the three success indicators used in this study show that if the defensive line on average is below a planning rate of 30 , it succeeds the attacking line no matter how flexible they play.

Concerning these findings, passing drills and coaching should be optimized to develop flexible decision-making for future professional football players.

Further research based on this study's results should examine the differences 
between flexibility rates of a four-man defensive line and a three- or five-man defensive line. The same should be done for different attacking line-ups.

\section{Conflicts of Interest}

The authors declare no conflicts of interest regarding the publication of this paper.

\section{References}

[1] Memmert, D. and Raabe, D. (2018) Data Analytics in Football: Positional Data Collection, Modelling and Analysis. Taylor \& Francis Inc., London.

https://doi.org/10.4324/9781351210164

[2] Wellman, M.P. (2006) Methods for Empirical Game-Theoretic Analysis. Association for the Advancement of Artificial Intelligence, Menlo Park, 1552-1556.

[3] Tuyls, K., Omidshafiei, S., Muller, P., Wang, Z., Connor, J., Hennes, D., et al. (2021) Game Plan: What AI Can Do for Football, and What Football Can Do for AI. Journal of Artificial Intelligence Research, 71, 41-88. https://doi.org/10.1613/jair.1.12505

[4] Lowe, Z. (2013) Lights, Cameras, Revolution.

[5] Maksai, A., Wang, X. and Fua, P. (2016) What Players Do with the Ball: A Physically Constrained Interaction Modeling. Proceedings of the IEEE Conference on Computer Vision and Pattern Recognition, Las Vegas, 27-30 June 2016, 972-981. https://doi.org/10.1109/CVPR.2016.111

[6] Le, H. M., Yue, Y., Carr, P. and Lucey, P. (2017) Coordinated Multi-Agent Imitation Learning. International Conference on Machine Learning, New York, 19-24 June 2016, 1995-2003.

[7] Le, H.M., Carr, P., Yue, Y. and Lucey, P. (2017) Data-Driven Ghosting Using Deep Imitation Learning. MIT Sloan Sports Analytics Conference, Boston, 3-4 March 2017, 1-15.

[8] Perl, J. and Memmert, D. (2018) Soccer: Process and Interaction. In: Baca, A. and Perl, J., Eds., Modelling and Simulation in Sport and Exercise, Routledge, Abingdon, 73-94. https://doi.org/10.4324/9781315163291-4

[9] Perl, J. and Memmert, D. (2018) Key Performance Indicators. In: Baca, A. and Perl, J., Eds., Modelling and Simulation in Sport and Exercise, Routledge, Abingdon, 146-166. https://doi.org/10.4324/9781315163291-8

[10] Memmert, D. and Rein, R. (2018) Match Analysis, Big Data and Tactics: Current Trends in Elite Soccer. German Journal of Sports Medicinel Deutsche Zeitschrift für Sportmedizin, 69, 65-72. https://doi.org/10.5960/dzsm.2018.322

[11] Perl, J. (2018) Formation-Based Modelling and Simulation of Success in Soccer. International Journal of Computer Science in Sport, 17, 204-215. https://doi.org/10.2478/ijcss-2018-0012

[12] Perl, J. Imkamp, J. and Memmert, D. (2021) Key Strictness vs. Flexibility: Simulation-Based Recognition of Strategies and Its Success in Soccer. International Journal of Computer Science in Sport, 20, 43-54. https://doi.org/10.2478/ijcss-2021-0003

[13] Perl, J. and Memmert, D. (2011) Net-Based Game Analysis by Means of the Software Tool Soccer. International Journal of Computer Science in Sport, 10, 77-84.

[14] Perl, J., Grunz, A. and Memmert, D. (2013) Tactics in Soccer: An Advanced Approach. International Journal of Computer Science in Sport, No. 12, 33-44. 
[15] Low, B., Coutinho, D., Gonçalves, B., Rein, R., Memmert, D. and Sampaio, J. (2019) A Systematic Review of Collective Tactical Behaviours in Football Using Positional Data. Sports Medicine, 50, 343-385. https://doi.org/10.1007/s40279-019-01194-7

[16] Perl, J. and Memmert, D. (2017) A Pilot Study on Offensive Success in Soccer Based on Space and Ball Control-Key Performance Indicators and Key to Understand Game Dynamics. International Journal of Computer Science in Sport, 16, 65-75. https://doi.org/10.1515/ijcss-2017-0005

[17] Memmert, D., Raabe, D., Schwab, S. and Rein, R. (2019) A Tactical Comparison of the 4-2-3-1 and 3-5-2 Formation in Soccer: A Theory-Oriented, Experimental Approach Based on Positional Data in an 11 vs. 11 Game Set-Up. PLoS ONE, 14, e0210191. https://doi.org/10.1371/journal.pone.0210191

[18] Low, B., Rein, R., Raabe, D., Schwab, S. and Memmert, D. (2021) The Porous High-Press? An Experimental Approach Investigating Tactical Behaviours from Two Pressing Strategies in Football. Journal of Sports Sciences, 1-12. (In Press) https://doi.org/10.1080/02640414.2021.1925424

[19] Baca, A., Dabnichki, P., Heller, M. And Kornfeind, P. (2009) Ubiquitous Computing in Sports: A Review and Analysis. Journal of Sport Science, 27, 1335-1346. https://doi.org/10.1080/02640410903277427

[20] Groom, R., Cushion, C. and Nelson, L. (2011) The Delivery of Video-Based Performance Analysis by England Youth Soccer Coaches: Towards a Grounded Theory. Journal of Applied Sport Psychology, 23, 16-32. https://doi.org/10.1080/10413200.2010.511422

[21] Rein, R., Perl, J. and Memmert, D. (2017) Maybe a Tad Early for a Grand Unified Theory: Commentary on "Towards a Grand Unified Theory of Sports Performance". Human Movement Science, 56, 173-175.

https://doi.org/10.1016/j.humov.2017.04.011 